\title{
Vesicular Glutamate Transporter 3 Is Required for Synaptic Transmission in Zebrafish Hair Cells
}

\author{
Nikolaus Obholzer, ${ }^{1}$ Sean Wolfson, ${ }^{1}$ Josef G. Trapani, ${ }^{1}$ Weike Mo, ${ }^{1}$ Alex Nechiporuk, ${ }^{2}$ Elisabeth Busch-Nentwich, ${ }^{3}$ \\ Christoph Seiler, ${ }^{3}$ Samuel Sidi, ${ }^{3}$ Christian Söllner ${ }^{3}$ Robert N. Duncan, ${ }^{1}$ Andrea Boehland, ${ }^{1}$ and Teresa Nicolson ${ }^{1}$ \\ ${ }^{1}$ Howard Hughes Medical Institute, Oregon Hearing Research Center and Vollum Institute, Oregon Health \& Science University, Portland, Oregon 97239, \\ ${ }^{2}$ Department of Biological Structure, University of Washington, Seattle, Washington 98195, and ${ }^{3}$ Max-Planck-Institut für Entwicklungsbiologie, 72076 \\ Tübingen, Germany
}

Hair cells detect sound and movement and transmit this information via specialized ribbon synapses. Here we report that asteroid, a gene identified in an ethylnitrosourea mutagenesis screen of zebrafish larvae for auditory/vestibular mutants, encodes vesicular glutamate transporter 3 (Vglut3). A splice site mutation in exon 2 of vglut3 results in a severe truncation of the predicted protein product and morpholinos directed against the vglut3 ATG start site or the affected splice junction replicate the asteroid phenotype. In situ hybridization shows that vglut 3 is exclusively expressed in hair cells of the ear and lateral line organ. A second transporter gene, vglut1, is also expressed in zebrafish hair cells, but the level of $v$ glut $1 \mathrm{mRNA}$ is not increased in the absence of Vglut 3 . Antibodies against Vglut 3 label the basal end of hair cells and labeling is not present in asteroid/vglut 3 mutants. Based on the localization of Vglut 3 in hair cells, we suspected that the lack of vestibulo-ocular and acoustic startle reflexes in asteroid/vglut 3 mutants was attributable to a defect in synaptic transmission in hair cells. In support of this notion, action currents in postsynaptic acousticolateralis neurons are absent in asteroid/vglut3 mutants. At the ultrastructural level, mutant asteroid/vglut3 hair cells show a decrease in the number of ribbon-associated synaptic vesicles, indicating a role for Vglut3 in synaptic vesicle biogenesis and/or tethering to the ribbon body. Lack of postsynaptic action currents in the mutants suggests that the remaining hair-cell synaptic vesicles contain insufficient levels of glutamate for generation of action potentials in first-order neurons.

Key words: vglut3; zebrafish; action currents; hair cells; synaptic vesicles; ribbon synapse; vglut 1

\section{Introduction}

Hair cells are the sensory receptors for the auditory and vestibular system. They possess a highly specialized structure, the synaptic ribbon, which coordinates synaptic vesicles (SVs) at the active zone. Synaptic ribbons confer remarkable release properties to the hair-cell afferent synapse: exocytosis (and subsequent replenishment) at a single synapse can occur at rates approaching 1000 SV/s (Moser and Beutner, 2000; Griesinger et al., 2005), and unlike conventional synapses, tens of thousands of SVs can be released from hair cells in response to strong stimulation (Schnee et al., 2005). It is estimated that frog saccular hair cells contain up to

\footnotetext{
Received July 5, 2007; revised Jan. 15, 2008; accepted Jan. 16, 2008.

This work was supported in part by National Institutes of Health Grants R01 DC006880 and PA-01-011. We thank Fangyi Chen for designing and building the VOR apparatus, Stephanie Kaech-Petri and Jacqueline DeGagne for their technical assistance with the confocal and electron microscopy, and Peter Mayinger for his comments on this manuscript. We also thank the members of the Tübingen 2000 Screen Consortium for making this work possible.

Correspondence should be addressed to Teresa Nicolson, Howard Hughes Medical Institute, Oregon Hearing Research Center and Vollum Institute, Oregon Health \& Science University, 3181 Southwest Sam Jackson Park Road, Portland, 0R 97239. E-mail: nicolson@ohsu.edu.

E. Busch-Nentwich's and C. Söllner's present address: Wellcome Trust, Sanger Institute, Wellcome Trust Genome Campus Hinxton, Cambridge CB10 1SA, UK.

C. Seiler's present address: Department of Medicine, University of Pennsylvania, 1230 Biomedical Research Building II/III, 421 Curie Boulevard, Philadelphia, PA 19104.

S. Sidi's present address: Department of Pediatric Oncology, Dana-Farber Cancer Institute, Harvard Medical School, Mayer Building 630, 44 Binney Street, Boston, MA 02115.

DOI:10.1523/JNEUROSCI.5230-07.2008

Copyright (C) 2008 Society for Neuroscience $\quad 0270-6474 / 08 / 282110-09 \$ 15.00 / 0$
}

200,000-600,000 clear core SVs (Lenzi et al., 1999). How this enormous pool of SVs is generated, tethered, and regulated is unknown.

Several lines of evidence indicate that the hair-cell afferent synapse is glutamatergic. EPSCs in afferent boutons are mediated by AMPA receptors (Glowatzki and Fuchs, 2002), supporting cells abutting the afferent synapse express the glutamateaspartate transporter (GLAST) (Furness et al., 2002), and phosphate-activated glutaminase (a glutamate synthetic enzyme) has been detected in the mitochondria of hair cells in the mammalian cochlea (Takumi et al., 1999). It was reported previously that vesicular glutamate transporter 3 (VGLUT3) immunoreactivity is present in the sensory epithelial cells and afferent fibers of the peripheral vestibular system in rats (Wang et al., 2007). Furthermore, mRNA for VGLUT1 has been isolated from cochlear hair cells, and at least one group has reported VGLUT1 protein at the guinea pig afferent synapse (Furness and Lawton, 2003).

Vertebrates possess three VGLUT isoforms. In the mature mammalian CNS, VGLUT1 and VGLUT2 appear in essentially nonoverlapping distributions, although they are coexpressed in some neurons during development (Fremeau et al., 2004a). VGLUT3 is found in scattered locations in the CNS of rats including the lateral superior olive and other auditory nuclei (Gillespie et al., 2005; Deng et al., 2007). It has also been detected in amacrine cells of the retina in rodents and humans (Johnson et al., 
2004; Haverkamp et al., 2004; Gong et al., 2006). Interestingly, VGLUT3 is expressed in a subset of serotonergic (Gras et al., 2005) and GABAergic neurons (Fremeau et al., 2001), and also in non-neuronal cells such as pancreatic islet cells, suggesting that glutamate may have novel modulatory and/or signaling roles (Takamori, 2006).

As part of a screening consortium, we isolated several new zebrafish auditory and vestibular mutants with defects in haircell function (our unpublished results). Mutant asteroid larvae have normal hair-bundle morphology and show wild-type levels of $\mathrm{N}$-(3-triethylammoniumpropyl)-4-(4-(dibutylamino)styryl)pyridinium dibromide (FM1-43) uptake (our unpublished results), indicative of normal mechanotransduction (Seiler and Nicolson, 1999; Gale et al., 2001). However, although they exhibit a normal touch-response, asteroid larvae appear to be deaf and display a profound balance defect, suggesting a defect acting downstream of mechanotransduction. We have positionally cloned the asteroid gene, and report here that it encodes the Vglut isoform, Vglut3. Absence of Vglut3 in asteroid mutants results in a reduction of the number of synaptic vesicles surrounding ribbon bodies in hair cells and abolishes postsynaptic action currents.

\section{Materials and Methods}

Molecular biology. Genomic DNA and mRNA were extracted from day 5 zebrafish larvae by standard methods. First-strand cDNA was generated with the SuperScript III First-Strand Synthesis System (Invitrogen, Carlsbad, CA) and an oligo-dT primer, and partial vglut3 ds-cDNA was generated by PCR (forward primer 5'-AGTCCAGTGCAGGAGAGGATGCG-3'; reverse primer 5-CGCACAAACATCACACAACCATAGTGAACC-3'). The GenBank accession number for full-length $v g l u t 3$ is pending.

Generation of neurod:egfp transgenic line. We modified a neurodcontaining bacterial artificial chromosome (BAC) clone by Escherichia coli-based homologous ET-recombination (Zhang et al., 1998). BAC clone $\mathrm{dK} 33 \mathrm{~b} 12$ contains $\sim 67 \mathrm{~kb}$ of sequence upstream and $89 \mathrm{~kb}$ downstream of neurod (http://www.sanger.ac.uk/Projects/D_rerio/mapping. shtml). After recombination, the modified BAC clone contained an enhanced green fluorescent protein (egfp) gene positioned at an endogenous start site. The accuracy of recombination was evaluated by PCR, sequencing, and by transient expression assays. To obtain a germline, we injected linearized BAC DNA into zebrafish embryos, raised injected fish to adulthood, and screened their progeny for reporter gene expression. The germline transmission rate was $0.4 \%$. egfp reporter faithfully recapitulated endogenous neurod expression in cranial ganglia as well as other organs. The neurod:egfp strain has been outcrossed for two generations and transmitted the transgene in a Mendelian manner.

In situ hybridization. Digoxygenin-labeled riboprobes were generated with the DIG Labeling Kit (Roche, Welwyn Garden City, UK) from fragments of vglut1 (nucleotides 1-692), vglut2a (nucleotides 1-797) and $b / c$ (nucleotides 789-2352), and vglut3 (nucleotides 1653-2445) cDNA that had been cloned into pCR-II (Invitrogen). In situ hybridization was conducted as described previously (Sollner et al., 2004) with $200 \mathrm{ng}$ of probe. Larvae at $72 \mathrm{~h}$ post fertilization (hpf) were imaged on a Zeiss (Oberkochen, Germany) Axio bright-field microscope using a $10 \times$ or $20 \times$ dry lens objective. Images were acquired via an AxioCam MRc5 color digital camera using Axiovision software and exported as TIFF files to Adobe (San Jose, CA) Photoshop for analysis.

Morpholino injection. Morpholinos against the vglut3 start codon "ATG MO" (5'-CCAGTGGCATCTCTCCTTCCCTTTC-3') and intron 2 splice-donor site "GT MO" (5'-ATTTATTTCTGGTTTCACCTGCATG$3^{\prime}$ ) as well as five-base mismatch controls "ATG control MO" and "GT control MO" were obtained from GeneTools (Philomath, OR) as lyophilized solids and resuspended in RNase-free double-distilled $\mathrm{H}_{2} \mathrm{O}(2 \mathrm{mM})$. Additional morpholinos include "VG1_GT MO" against the vglut1 intron 2 splice-donor site (5' -CAGCACTGATACTGACCACTATGAC-3') as well as the inverted control. Injection solutions were made by diluting these stocks to final concentrations of $500 \mu \mathrm{M}$ for MOs, in $100 \mathrm{~mm} \mathrm{KCl}$. Phenol red was added to a final volume of $5 \%$ as an injection tracer. Morpholinos were injected into the yolk of one-cell stage embryos on the day of collection, and larvae were raised in $\mathrm{E} 3$ embryo medium at $30^{\circ} \mathrm{C}$ until screening at day 4 .

Immunohistochemistry and fluorescence imaging. Affinity-purified rabbit polyclonal antibodies against the $\mathrm{C}$ terminus (amino acids 530-590) of Danio rerio Vglut 3 and the N-terminal portion (amino acids 133-483) of $D$. rerio Ribeye b were generated by Proteintech (Chicago, IL). An anti-acetylated tubulin antibody was obtained from Sigma (St. Louis, MO). Immunohistochemistry was performed on $14-\mu \mathrm{m}$-thick cryosections and whole-mount larvae following standard sectional immunostaining procedures and using fish gelatin blocking buffer. For imaging, larvae were mounted in Antifade reagent (Invitrogen) and imaged with an Olympus (Tokyo, Japan) FluoView 300 line-scanning confocal head attached to an Olympus BX51 upright microscope using a $60 \times(1.4$ numerical aperture) oil-immersion objective and $4 \times$ electronic zoom. A total of three neuromasts and several neuroepithelia of the inner ear were imaged per specimen of sibling larvae $(n=8)$ and mutant larvae $(n=8)$. Excitation wavelengths of $488 \mathrm{~nm}$ (Alexa488 conjugated) and $543 \mathrm{~nm}$ (Cy3-conjugated secondary antibodies) were applied, and emitted light was collected through $510-530 \mathrm{~nm}$ and $605 \pm 12.5 \mathrm{~nm}$ barrier filters, respectively.

The plasmid containing vglut1, pEGFP-N1 cytomegalovirus (CMV) M6BP [myosin $6 b(\mathrm{M} 6)$ ], is a modified version of pEGFP-N1 (Clontech, Mountain View, CA) in which the CMV promoter was excised using the flanking AseI and NheI sites. These sites were subsequently filled in using Klenow fragment, polished using $p f u$ polymerase, and ligated, thus generating the intermediate pEGFP-N1 CMV. Approximately $6.5 \mathrm{~kb}$ of the myo6b promoter were inserted into the multiple cloning site using SacI and EcoRI sites. For expression of growth-associated protein 43 (GAP43), the plasmid myo6b:gap43-tdtomato was generated using the same promoter driving the expression of tandem dimer (td) DsRed fluorescent protein "tomato," a gift from Roger Y. Tsien (University of California, San Diego, La Jolla, CA) (Shu et al., 2006). tdtomato, was fused at its $\mathrm{N}$ terminal to the first 20 amino acids of zebrafish GAP43. This sequence is palmitoylated at the cysteines in positions 3 and 4 and targets the protein to the membrane (Zuber et al., 1989).

Measuring the vestibulo-ocular reflex and the optokinetic response. The compensatory eye movement is defined as the vestibulo-ocular reflex (VOR) when angular or linear accelerations are detected by the vestibular system. We designed a VOR apparatus to measure the angular and linear VOR in zebrafish by rotating the larva in the yaw axis with the animal positioned perpendicular to the horizontal plane (supplemental Fig. 1, available at www.jneurosci.org as supplemental material). Five-day-old zebrafish larvae were placed inside a drop of $4 \%$ methyl cellulose or $1.5 \%$ low-melting agarose in the center of a cover glass with the dorsal side up. The cover glass was put onto the vertical sample platform with the larval head facing downward. To stimulate the larva, the turntable was driven by a motor at the frequency of $45^{\circ} / \mathrm{s}$ and amplitude of $\pm 45^{\circ}$. The direction of movement was controlled manually by a reversing switch. Meanwhile, eye movements were recorded by a digital camera (DCM300; Hangzhou Scopetek OptoElectric, Zhejiang, China) through a $10 \times$ objective lens (Mitutoyo, Neuss, Germany), illuminated by an infrared ( $800 \mathrm{~nm}$; illumination control) or a normal led light source, at 3-5 frames per second and $1024 \times 768$ dpi resolution per frame. Positions of the rotation platform were illustrated by using a laser point shining on the sample platform at a given position and recorded by the digital camera simultaneously, which allowed us to read the platform position from the video directly.

Frames were extracted from the video with 5-10 rotation cycles for additional analysis. Within each frame, the shortest distance between a single iris and body axis was measured manually using Photoshop software. To account for any tracking errors, we followed a particular landmark on the head such as a clearly visible melanocyte and measured its change in position during rotation. Random relative movements of the melanocytes did not differ in amplitude $(0.0-0.05 \mathrm{~mm})$ between larvae.

The optokinetic response was measured by placing a larva in methyl cellulose on a coverslip inside an illuminated rotating drum with 11-mm-wide black and white stripes rotating at $20.5 \%$ s. Eye movements were recorded with an identical camera as above mounted on a dissecting scope. 


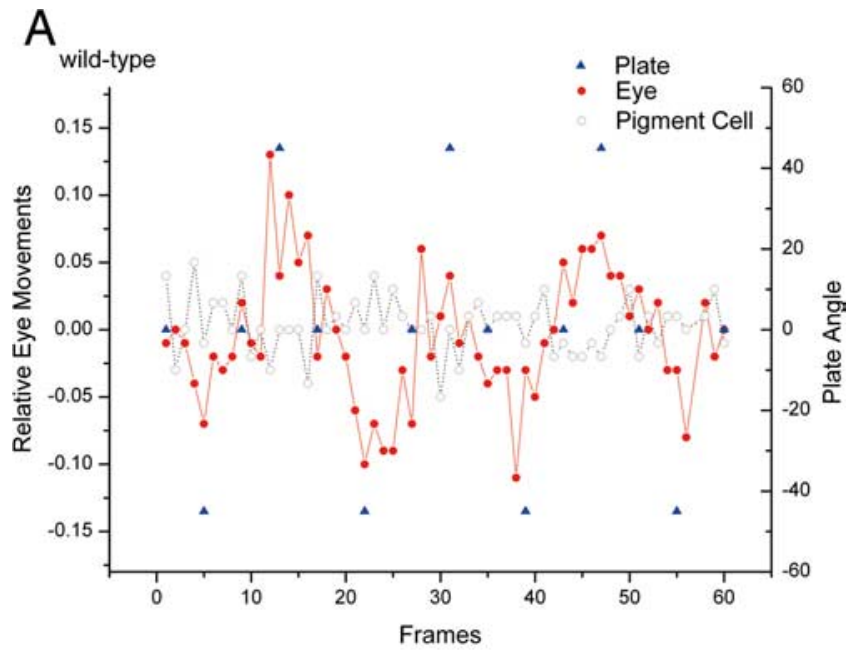

B

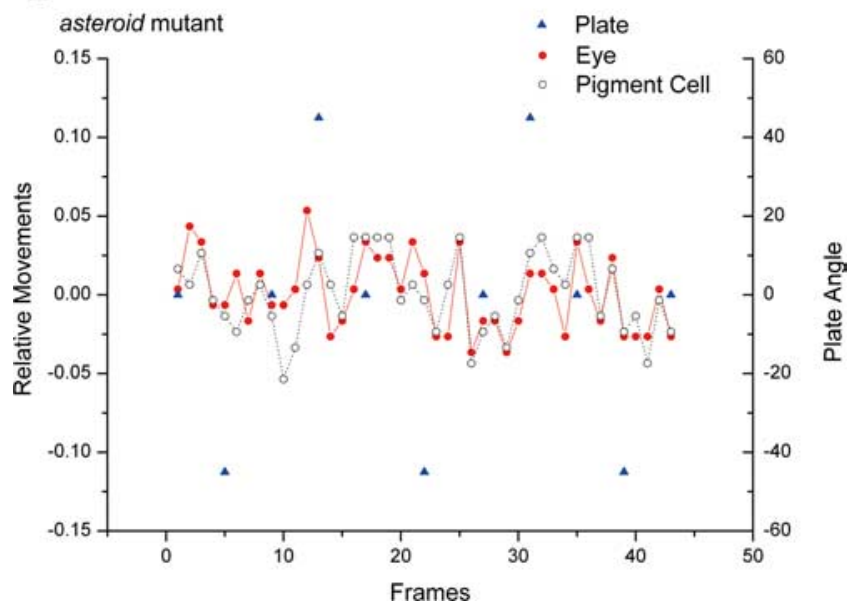

Figure 1. The vestibulo-ocular reflex is defective in mutant asteroid larvae. Representative eye-movement traces are shown in response to $45^{\circ}$ movements in the yaw axis at an angular velocity of $45^{\circ} / \mathrm{s}$. A, Relative eye movements in a given video frame of a sibling larva (red dots) coordinating with the angular position of the platform (blue triangles). $\boldsymbol{B}$, Irregular and reduced eye movements in an mutant asteroid larva. Movements were $0.5 \mathrm{~mm}$ in response to the rotation of the platform. The gray dots mark the relative motion of a pigment cell in each animal.

Microphonic and action current recordings. Day 5 larvae were anesthetized with $0.02 \% 3$-aminobenoic acid ethyl ester, (MESAB; Sigma) in normal extracellular solution and pinned on their side to a Sylgard-lined recording chamber (Siskiyou, Grants Pass, OR). To suppress muscle activity, larvae were microinjected with $125 \mathrm{~mm} \alpha$-bungarotoxin into the heart. Next, larvae were rinsed and left in normal extracellular solution [containing (in mM) $120 \mathrm{NaCl}, 2 \mathrm{KCl}, 2 \mathrm{CaCl}_{2}, 1 \mathrm{MgCl}_{2}$ and $10 \mathrm{HEPES}$, $\mathrm{pH}$ 7.3] for at least $5 \mathrm{~min}$ to recover from a potential block of mechanotransduction by MESAB. The recording chamber was then mounted to an upright microscope (Leica, Nussloch, Germany; DMLFS) equipped with differential interference contrast and epifluorescence, a $40 \times$ waterimmersion objective and an Orca-ER CCD camera (Hamamatsu, Hamamatsu, Japan). The recording electrode and water jet were positioned (see supplemental Fig. 3, available at www.jneurosci.org as supplemental material) using stepper-motor micromanipulators (Sutter Instruments, Novato, CA). The water jet was positioned $\sim 100 \mu \mathrm{m}$ from a given neuromast and displacement of the cupula was verified by eye. For microphonic potentials, recording micropipettes were positioned adjacent to the base of the cupula in the same plane as the hair cell bundles. The collection of extracellular action currents required micropipettes to be within the membrane sac that separates the posterior lateral line ganglion from the rest of the larvae. Access to the inside of the sac was obtained by increasing pressure of the recording pipette against the sac
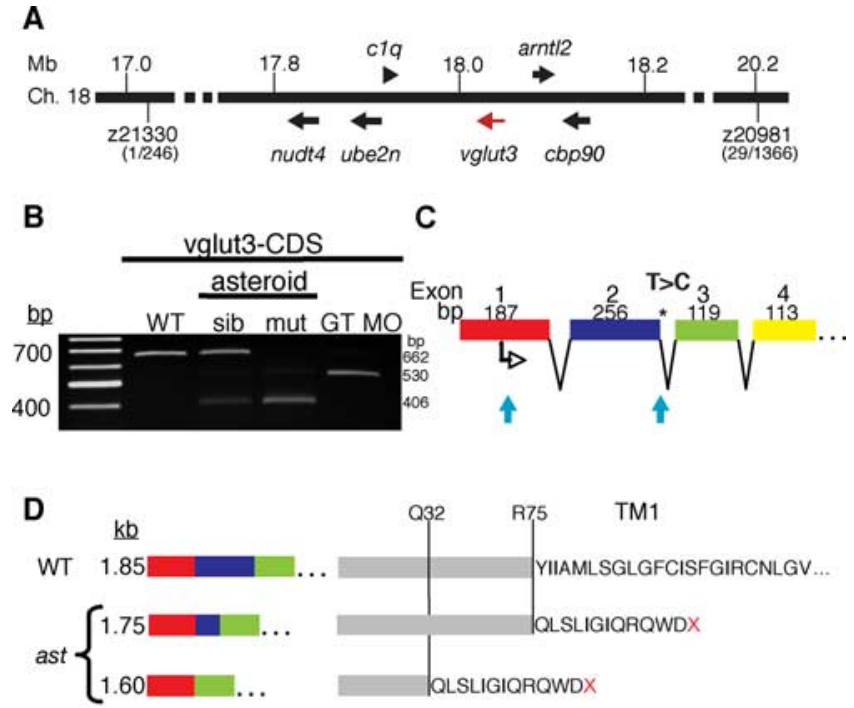

E

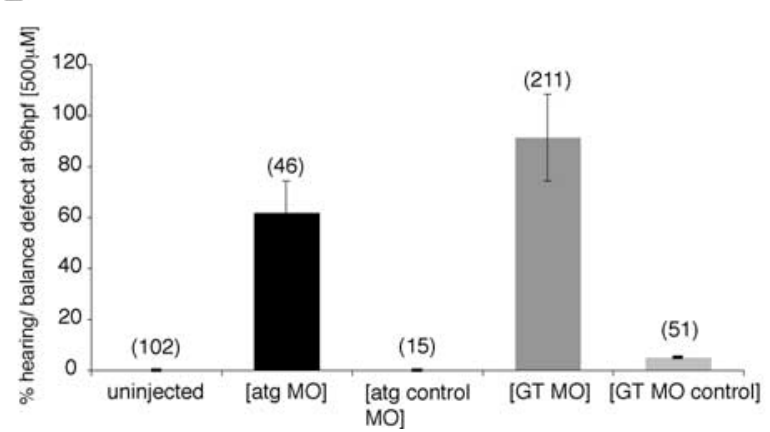

Figure 2. Positional cloning and identification of the asteroid gene. $A$, Critical interval of the asteroid gene, including polymorphic markers and the number of meiotic recombinants at each end. $\boldsymbol{B}$, CDNA spanning exon 2 of vglut3 amplified from wild-type, asteroid sibling and mutant, and vglut3 splice donor site (GT) morpholino-injected larvae. C, Exon/intron structure of vglut3, and position of the genomic lesion (asterisk), a GT $>\mathrm{GC}$ donor splice site mutation (blue arrows indicate the morpholino target sites in the transcript). $\boldsymbol{D}$, Reading frames of the wild-type and mutant transcripts. E, ATG and splice site (GT) morpholinos phenocopied the asteroid/vglut3 mutant. The percentage of larvae with auditory/vestibular defects is as follows: uninjected, $0 \pm$ 0; ATG M0, $62 \pm 13$; ATG control M0, $0 \pm 0 ; \mathrm{GT} M 0,91 \pm 12 ;$ GT control M0, $0 \pm 0$ ( $p \ll$ 0.001 , Student's $t$ test). Error bars denote SD. Number of total larvae observed in parentheses.

until the membrane gave way. Verification of pipette entry was by application of positive holding pressure and visualization of sac "inflation." Once inside, we observed random spikes and moved the pipette slightly to press against a particular cell body. After settling against the soma, we then searched for an innervated neuromast, choosing among the first four to five rostral neuromasts. Neuromasts were chosen for recordings when we observed the elimination of spontaneous firing and the persistence of phase-locking to the stimulus.

Mechanical stimulation. Lateral line hair cells were mechanically stimulated as described previously (Nicolson et al., 1998). Briefly, a pressure clamp (HSPC-1; ALA Scientific, Westbury, NY) attached to a glass micropipette (tip diameter, $\sim 30 \mu \mathrm{M}$ ) filled with normal extracellular solution was used to stimulate lateral line hair cells. The pressure clamp was driven by a sinusoidal voltage command that resulted in bidirectional deflection of the cupula of an individual neuromast by approximately $\pm 20^{\circ}$. To elicit activation of hair cell transduction channels, the water jet was oriented along the long body axis deflecting the cupula laterally. Note that displacement perpendicular to the long body axis did not yield any measurable change in potential. In addition, saturating concentrations of amiloride blocked completely all microphonic potentials and extracellular action currents.

Electrophysiology. For all experiments, both the microphonic potential and extracellular current recording micropipettes were filled with nor- 

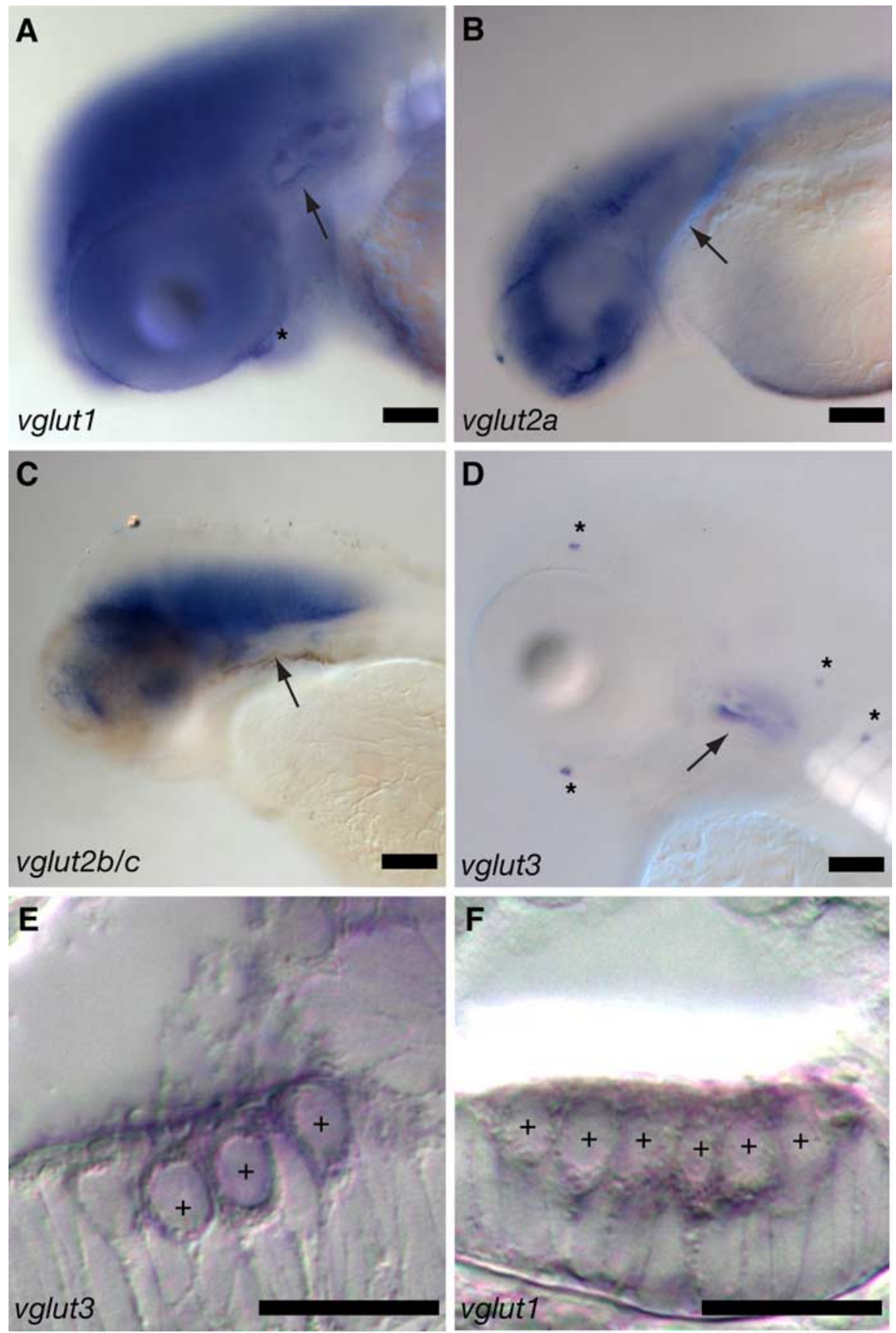

Figure 3. $\boldsymbol{A}-\boldsymbol{D}$, vglut expression in the developing zebrafish. mRNA (blue) was detected at $72 \mathrm{hpf}$ by whole-mount in situ hybridization with vglut1 $(\boldsymbol{A})$, vglut2a $(\boldsymbol{B})$, vglut $2 b$ and vglut $2 \boldsymbol{C}(\boldsymbol{C})$, and vglut $3(\boldsymbol{D})$ antisense riboprobes. $\boldsymbol{A}-\boldsymbol{F}$, Lateral views of the larval head region. Asterisks indicate labeled neuromasts. The arrows indicate the otic capsule in each specimen. $\boldsymbol{E}, \boldsymbol{F}$, Cryostat thick sections of the inner-ear hair cells in vglut3- and vglut7-labeled larvae, respectively. Plus signs mark hair-cell bodies. Scale bars: $A-D, 100 \mu \mathrm{m} ; \boldsymbol{E}, \boldsymbol{F}, 10 \mu \mathrm{m}$.

mal extracellular solution. The pipettes were fabricated from borosilicate glass (P-97; Sutter Instruments) and had tip diameters of $\sim 3$ and $1 \mu \mathrm{m}$, respectively. Pipette resistance ranged from 3 to $5 \mathrm{M} \Omega$ for microphonic electrodes and 12 to $15 \mathrm{M} \Omega$ for extracellular current electrodes. Signals were collected with an Axopatch 200B amplifier, a Digidata 1440A analog-to-digital board and pClamp 10 software (Molecular Devices, Sunnyvale, CA). Microphonic potentials were measured in current clamp mode and sampled at $100 \mu \mathrm{s} / \mathrm{pt}$. An additional amplifier (Brownlee, Santa Clara, CA; model 440) further amplified (total 50,000×) and filtered $(50 \mathrm{~Hz}$, eight-pole Bessel) the voltage signal. When necessary, a $60 \mathrm{~Hz}$ software driven notch filter (Clampex 10.0) was used to cancel out persistent line noise. The baseline was manually adjusted to zero for all traces of each recording. Extracellular currents were collected in voltage clamp mode, filtered at $1 \mathrm{kHz}$ and sampled at $100 \mu \mathrm{s} / \mathrm{pt}$. Data were analyzed with Clampfit 10 (Molecular Devices) and SigmaPlot 10 software (Systat Software, Point Richmond, CA).

Electron microscopy and vesicle counting. Day 5 asteroid mutants and siblings $(n=5$ specimens) were fixed overnight in 3\% glutaraldehyde and $1.5 \%$ paraformaldehyde in $0.1 \mathrm{M}$ phosphate buffer, stained with $1 \%$ osmium, dehydrated in ethanol and embedded in Araldite. An average of 11 transverse serial sections (70-90 nm thin sections) through the ear of each specimen was imaged on a Phillips (Eindhoven, The Netherlands) CM100 electron microscope. Each section contained at least one hair cell with a ribbon synapse. Hair-cell ribbons in the ear and neuromasts were imaged at $21,000-39,000 \times$ magnification. Images were acquired on an analog camera and negatives were scanned into Adobe Photoshop at 1200 dpi. Image levels were adjusted as necessary to reveal synaptic vesicles close to the ribbon bodies. Vesicles were then counted as described in the text.

\section{Results}

\section{The mutant asteroid phenotype}

Mutant asteroid larvae display a classic circler phenotype: no startle response to dish-tapping, an inability to orient to gravity, and a spiraling swim trajectory. The mutation is recessive and lethal, leading to death around $9 \mathrm{~d}$ postfertilization. To characterize the vestibular defects in asteroid mutants, we tested the VOR in 5-d-old homozygous larvae. This reflex occurs in response to head movements and promotes stabilization of images on the retina. When rotated at $45 \%$ s along the yaw axis while positioned horizontally (ventral side down), we did not observe a response in wild-type larvae, in agreement with a previous report (Beck et al., 2004). However, when positioned vertically (head facing down) and rotated at the same speed, we observed a pronounced increase in eye movements in response to head movements (Fig. 1A) $(n=12)$. This pronounced response may be caused by stimulation and activity of macular organs instead of ampullary organs, which appear to be nonfunctional at this stage (Beck et al., 2004). In contrast to wild-type siblings, mutant asteroid larvae showed drastically reduced eye movements that appeared randomly with respect to the position of the rotating platform (Fig. $1 B)(n=15)$. If we measured the movement of a clearly delineated melanocyte on the head, we found that the amplitude of relative movement of this landmark was comparable with that seen with mutant eyes (Fig. $1 B$ ). Unlike the VOR response in wild-type sibling larvae, the eye movements in asteroid mutants were not discernable with respect to the stimulus. Similar weak random eye movements were also seen in mutant cadherin23 larvae (tj264a allele) (data not shown). Compel- 
ling evidence suggests that Cadherin 23 is a component of the hair-cell tip link (Söllner et al., 2004, Siemens et al., 2004, Kazmierczak et al., 2007) and mutant cadherin23 larvae completely lack hair-cell receptor potentials (Nicolson et al., 1998). Thus, mutant asteroid larvae behave similarly to a mechanotransduction mutant with respect to their VOR response, suggesting that the eye movements are truly random and not caused by signaling from the auditory-vestibular system.

To rule out a visual defect or an inability to move the eyes in asteroid mutants, we tested the optokinetic response. When presented with a pattern of moving stripes, mutant asteroid larvae showed a robust response of eye movements tracking the stripes as seen in wild-type siblings $(n=3)$ (data not shown). This result suggests that vision is unaffected in asteroid mutants and that the larvae are not responding to visual cues during VOR tests. Testing the VOR in the dark using infrared light yielded the same result (data not shown).

FM1-43 uptake in mutant asteroid hair cells is indistinguishable from wild-type levels, suggesting that mechanotransduction is normal (data not shown). Measurements of receptor potentials in lateral line hair cells revealed no differences in the amplitude of electrical signals between siblings and asteroid mutants (see Fig. 6A) $(n=3)$. In addition, there were no detectable morphological defects in the inner ear or in the lateral line hair cells (data not shown). Together, these observations suggest that the asteroid defect lies downstream of mechanotransduction, perhaps in neurotransmission.

\section{Positional cloning of the asteroid gene}

Meiotic mapping with polymorphic markers tested on 1612 homozygous mutant larvae resolved a $\sim 5 \mathrm{Mb}$ critical interval that contained several predicted genes (Fig. 2A). Among these candidate genes was vglut3, which encodes an isoform of the vesicular glutamate transporter. PCR of the $5^{\prime}$ coding domain sequence of $v g l u t 3 \mathrm{cDNA}$ from wild-type and asteroid mutant larvae returned three amplicons: a 662 bp WT band, and 530 and 406 bp mutant bands from which exon 2 has been partially or completely deleted (Fig. 2 B). All three bands were recovered from asteroid sibling cDNA, indicating that the asteroid mutation may cause a splicing defect in vglut3 mRNA. Indeed, sequencing of the splicedonor site in intron 2 of the asteroid vglut3 gene revealed a GT $>$ GC transition. This mutation is expected to lead to the skipping or partial deletion of exon 2 during mRNA splicing (Fig. 2C). An antisense morpholino oligonucleotide (GT MO) directed against the intron 2 splice-donor site causes partial deletion of exon 2 that replicated the mutant asteroid auditory/vestibular phenotype (Fig. $2 B, E$ ). The predicted transla-
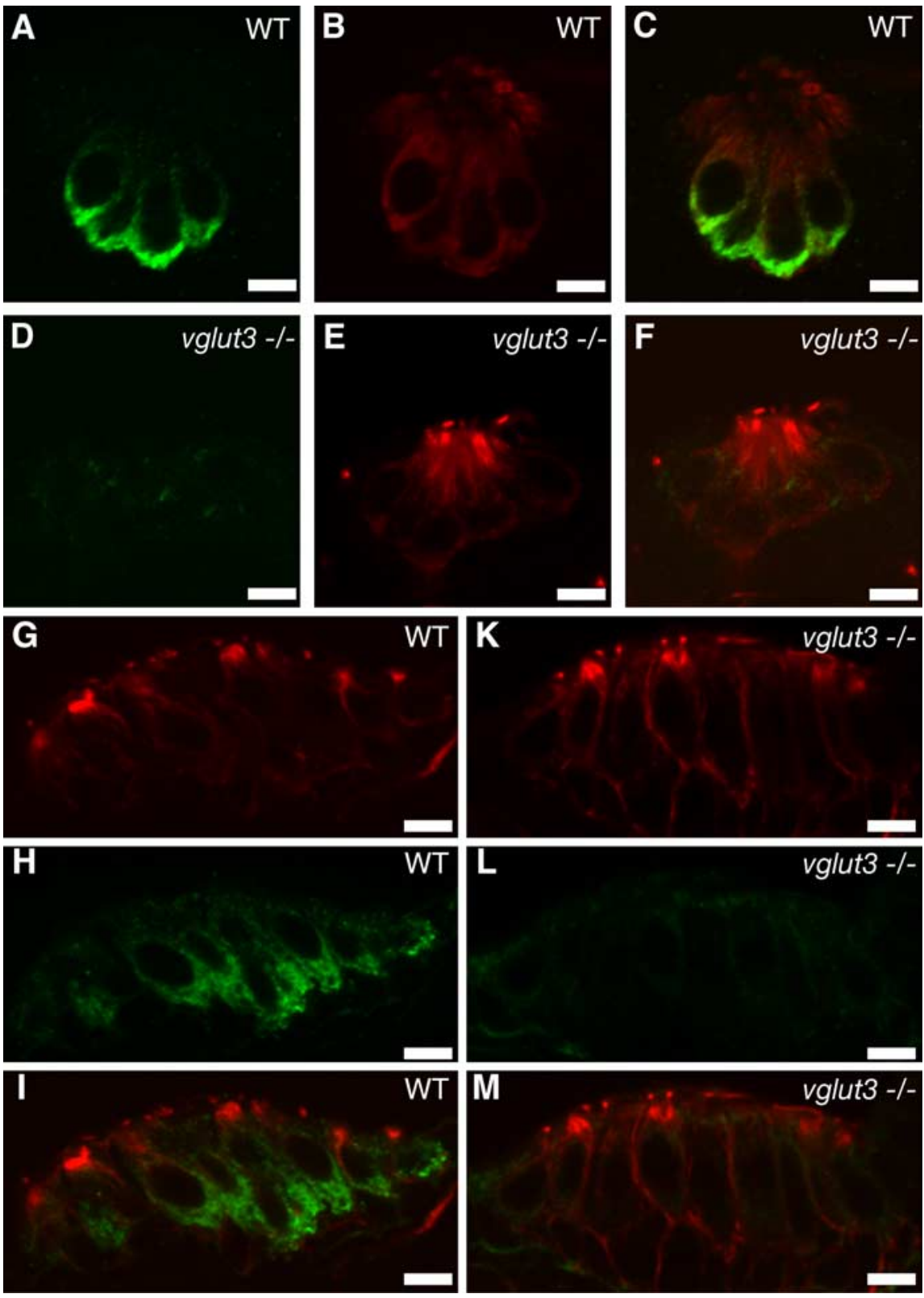

Figure 4. Vglut3 protein is absent in asteroid/vglut3 mutants. $\boldsymbol{A}-\boldsymbol{F}$, Immunolabeling of Vglut3 protein in thick sections of wild-type (wt) and mutant asteroid/vglut3 neuromast hair cells at $120 \mathrm{hpf}$. $\boldsymbol{A}-\boldsymbol{C}$, Thick section of a wild-type neuromast. $\boldsymbol{D}-\boldsymbol{F}$, hick section of a mutant asteroid/vglut 3 neuromast. $\mathbf{G}-\mathbf{M}$, Immunolabeling of macular hair cells in the inner ear at $120 \mathrm{hpf}$. G-I, Thick section of a wild-type macula. $\boldsymbol{K}-\boldsymbol{M}$, Thick section of a mutant asteroid/vglut3 macula. $\boldsymbol{A}, \boldsymbol{D}, \boldsymbol{H}, \boldsymbol{L}, \mathrm{Vglut} 3$ labeling (green). $\boldsymbol{B}$, $\boldsymbol{E}, \boldsymbol{G}, \boldsymbol{K}$, Acetylated tubulin staining to outline cell bodies (red). $\boldsymbol{C}, \boldsymbol{F}, \boldsymbol{I}, \boldsymbol{M}$, Overlays of tubulin and Vglut3 labeling. Scale bars: $3 \mu \mathrm{m}$.

tions of the cDNAs from asteroid and GT MO-morphants showed that both splicing defects result in a frameshift and premature stop codon that would terminate Vglut3 protein before its first transmembrane segment (Fig. 2D). Aside from the GT MO, another MO directed against the start codon (ATG MO) also replicated the asteroid phenotype, whereas five-base mismatch control MOs had no effect (Fig. $2 E$ ). We therefore conclude that vglut 3 is the asteroid gene, and that the asteroid mutation is functionally null.

\section{vglut 3 is exclusively expressed in hair cells}

To confirm our cloning results, we performed in situ hybridization for the three zebrafish vglut isoforms. Vglut1 showed a widespread distribution in the brain, and was also present in 

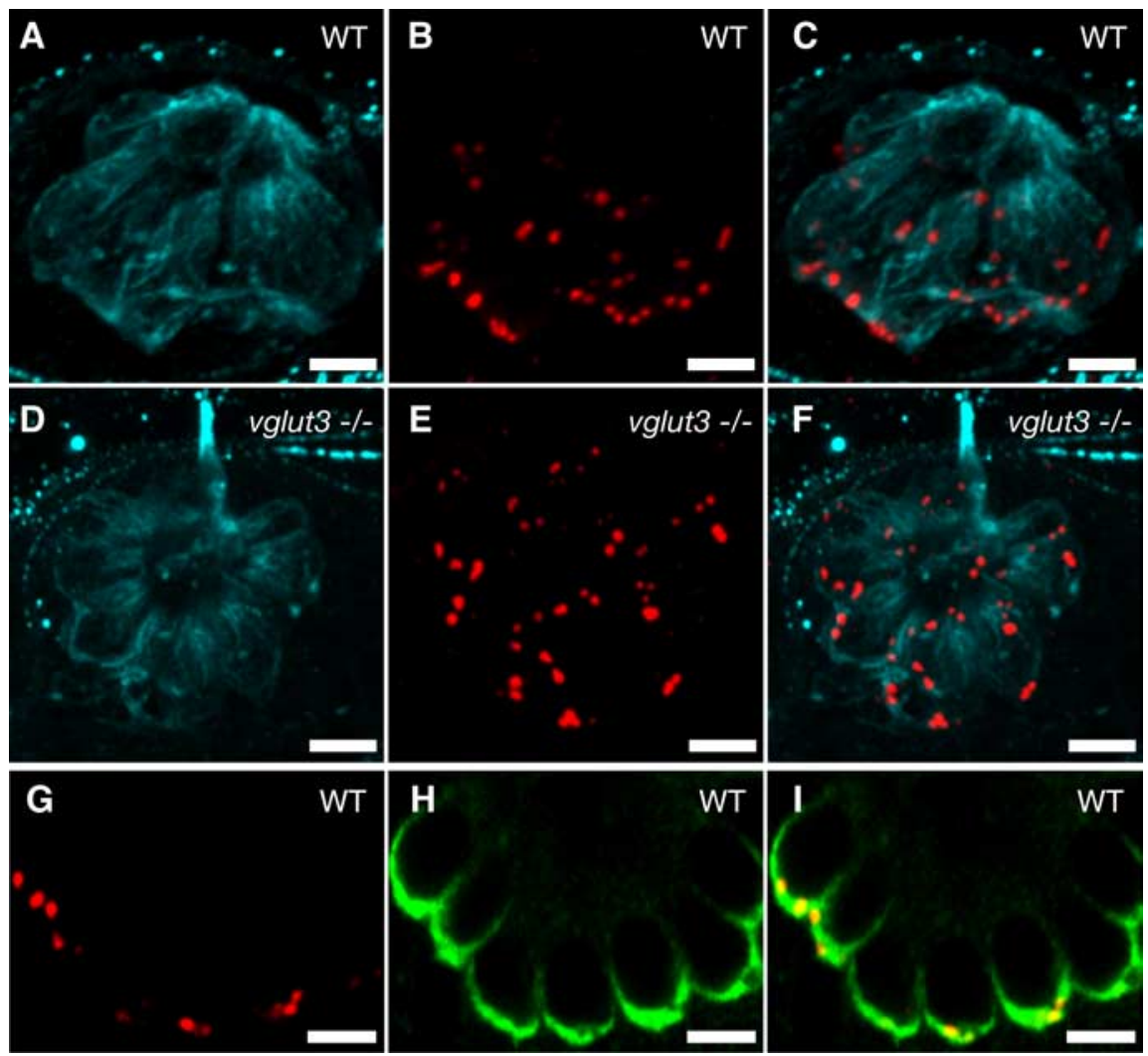

Figure 5. Ribbon bodies are normal in mutant asteroid/vglut3 larvae. $\boldsymbol{A}-\boldsymbol{F}$, Immunolabeling of Ribeye b in wild-type $(\boldsymbol{A}-\boldsymbol{C})$ and mutant $(\boldsymbol{D}-\boldsymbol{F})$ hair cells. Ribeye b stain is shown in red $(\boldsymbol{B}, \boldsymbol{E})$, whereas acetylated tubulin is shown in cyan blue to indicate the hair-cell bodies $(\boldsymbol{C}-\boldsymbol{F})$. $\mathbf{G}-\boldsymbol{I}$, Ribeye b (red) immunofluorescence colocalizes with Vglut3 labeling (green). Images are of a single confocal section $(0.5 \mu \mathrm{m})$ of a neuromast. Scale bars: $3 \mu \mathrm{m}$.

the larval inner ear and neuromasts (Fig. $3 A, F$ ). The three isoforms of $v g l u t 2$ were also present in the brain, but are absent from the inner ear (Fig. $3 B, C$ ). vglut3 expression, however, was detected only in the inner ear and in the neuromasts of the lateral line organ (Fig. $3 D, E$ ). Additional sectioning of the heads of labeled larvae did not reveal staining in neurons of the brain or eyes (data not shown). Thick sections of the inner ear (Fig. 3E,F) showed that vglut1 and vglut3 transcripts are present in hair cell somas.

Transient coexpression of VGLUT1 and VGLUT2 has been reported in mice (Wojcik et al., 2004, Fremeau et al., 2004). In Vglut1 knock-out mice, expression of VGLUT2 is not upregulated. We also did not observe increased levels of vglut $1 \mathrm{mRNA}$ in asteroid mutants (supplemental Fig. $2 A-E$, available at www.jneurosci.org as supplemental material). In addition, injection of morpholinos targeting the exon 2 splice site of vglut 1 caused deafness and balance defects in free swimming larvae, although the efficacy of the vglut $1 \mathrm{MO}$ was less striking (supplemental Fig. $2 \mathrm{~F}$, available at www.jneurosci.org as supplemental material). Aside from auditory/vestibular defects, vglut1 morphants had additional defects (smaller eyes, less active or responsive to touch), probably reflecting the widespread distribution of this transporter throughout the brain and retina.

These results suggest that, whereas vglut1 is expressed in hair cells of the inner ear and plays a broader role in the nervous system, vglut3 is a hair-cell specific vesicular glutamate transporter. The data further suggest that under our experimental conditions neither Vglut1 nor Vglut3 is able to compensate for the loss of the other in asteroid mutants or vglut1 morphant animals, respectively.
Vglut3 is localized to the basal end of hair cells

To examine the subcellular distribution of Vglut3, we labeled thick sections of larval fish with an affinity purified antibody against the zebrafish $\mathrm{C}$ terminus (amino acids 530-590) of Vglut3 (Fig. 4). For outlining the cell bodies of hair cells, we colabeled with an antibody against acetylated tubulin. As expected for a synaptic vesicle protein, Vglut3 immunoreactivity was present in the basal halves of inner ear and neuromast hair cells (Fig. 4A, $H$ ). Preincubation with the peptide antigen eliminated labeling in wild-type hair cells (data not shown). Moreover, Vglut3 immunoreactivity was not detectable above background labeling in asteroid mutants (Fig. $4 D, L$ ), confirming the identity of vglut 3 as the asteroid gene. Immunoreactivity was also not detected in sections of larval eyes and brain (data not shown).

Commercial antibodies against VGLUT1 did not show cross-reactivity in zebrafish hair cells (data not shown). In lieu of an antibody for Vglutl protein, we fluorescently tagged Vglut1 and transiently expressed Vglut1-GFP in hair cells using a hair-cell specific myosin $6 b$ promoter. As seen with Vglut3 immunoreactivity, Vglut1-GFP was largely confined to the basal end of hair cells (supplemental Fig. 2G-I, available at www.jneurosci.org as supplemental material).

In mice, coexpressed VGLUT isoforms can segregate to separate synapses within a given neuron (Fremeau et al., 2004b). To determine whether Vglut3 localizes to all synaptic ribbons within a hair cell or to a particular subset of ribbon bodies, we colabeled hair cells with anti-Vglut 3 antibody and an affinity purified polyclonal antibody against the $\mathrm{N}$ terminus of Ribeye b protein (Fig. 5). Ribeye protein is thought to constitute the bulk of the ribbon's mass (Schmitz et al., 2000). Immunoreactivity with anti-Ribeye antibody was localized to the basal end of hair cells in dense, large punctae (Fig. $5 B$ ). In neuromasts, each hair cell had two to five such structures. No obvious differences in number, shape or size of the labeled punctae was observed in wild-type versus mutant larvae (Fig. $5 A-F$ ). In single optical sections (500 $\mathrm{nm}$ thick), colabeling with anti-Vglut 3 antibody showed immunoreactivity associated with each of the Ribeye immunoreactive structures (Fig. $5 G-I)$. These results suggest that at the light microscopic level, Vglut 3 appears to be present at all ribbon synapses in hair cells, rather than a subset of synapses as seen in the CNS neurons.

\section{Postsynaptic action currents are absent in asteroid/vglut3 mutants}

Exclusive expression of Vglut 3 in hair cells suggests that a defect in hair-cell synaptic transmission could account for the vestibular/auditory phenotype in asteroid/vglut3 mutants. We therefore developed a preparation that would allow us to assess synaptic transmission in undissected, intact animals. To identify the ganglion, we used a neurod:GFP transgenic line that expresses GFP in the posterior lateral line nerve (supplemental Fig. 3, available at 
A WT

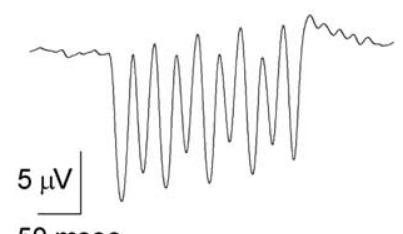

$50 \mathrm{msec}$
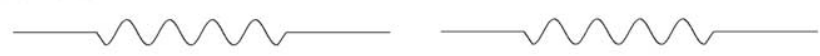

B

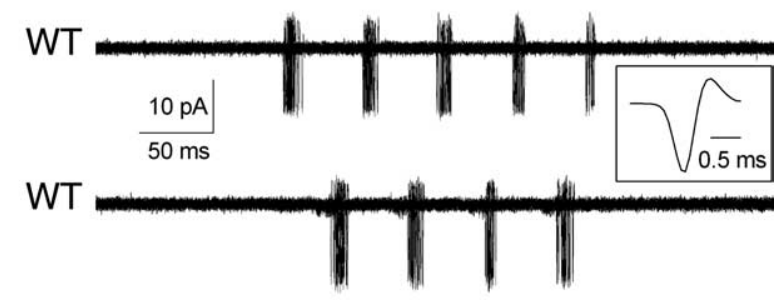

vglut3 -/-

\section{$20 \mathrm{~Hz}$}

Figure 6. Normal hair-cell receptor potentials and absent action currents in mutant asteroid/vglut3 larvae. A, Microphonic potentials from day 5 asteroid/vglut3 wild-type sibling and mutant larvae. Top traces are the average voltage response (200 presentations) to a $20 \mathrm{~Hz}$ water-jet stimulus (bottom traces) from a sibling and mutant larva, respectively. $\boldsymbol{B}$, Extracellular currents recorded from the posterior lateral line ganglion during $20 \mathrm{~Hz}$ stimulation of a lateral line neuromast. Top, Fifty consecutive sweeps containing 163 action currents phase locked to one direction of deflection. Inset, Signal average of all 163 events. Middle, Fifty consecutive traces from the sibling wild-type larva in $\boldsymbol{A}$ (note that the 88 action currents are phase locked to the opposite direction of deflection compared with the top trace). Bottom, Fifty consecutive current records from the asteroid/vglut3 mutant in $\boldsymbol{A}$.

www.jneurosci.org as supplemental material). We found that mechanical stimulation of lateral line hair bundles led to action currents that could be recorded extracellularly at the cell bodies within the posterior lateral line ganglion (Fig. $6 B$, supplemental Fig. 3, available at www.jneurosci.org as supplemental material). Stimulus-dependent spikes were immediately abolished by (1) introduction of a blocker of mechanotransduction, amiloride in the bath, (2) movement of the water jet 20 micrometers away from the neuromast, (3) moving the recording pipette several micrometers away from the ganglion, or (4) biasing the stimulus toward one direction (data not shown) (see below). The action currents were phase locked to the stimulus and indicated that each neuron innervated a single population of hair cells oriented in one direction within the neuromast (Fig. $6 B$ ). In Figure $6 B$, the top traces (50 consecutive sweeps) are from one wild-type cell that responded when the cupula of a neuromast was deflected caudally. The middle traces are from a wild-type cell that synapsed with hair cells that responded to deflections toward the head. Action currents with similar amplitudes $(21.2 \pm 3.5 \mathrm{pA})$ and wave form (Fig. $6 \mathrm{~B}$, inset) were detected in all siblings tested $(n=12)$. In addition, spontaneous action currents of similar amplitude and waveform were always observed in siblings (data not shown). For these experiments siblings were either homozygous wild-type or heterozygous. Genotyping of a sibling confirmed that spikes were present in heterozygous animals. In contrast, no currents were observed in asteroid/vglut3 mutants after mechanical stimulation of any individual neuromast along the trunk (Fig. 6C, bottom trace) $(n=11)$. Furthermore, extensive probing of the entire ganglion in mutants, which was not required to observe currents in wild-type larvae, never resulted in detectable action currents, spontaneous or otherwise.

\section{Synaptic vesicle numbers are reduced in asteroid/vglut 3 mutants}

A previous study of vglut1 knock-out mice reported a $\sim 60 \%$ reduction in reserve pool SVs at central synapses (Fremeau et al. 2004). We speculated that a similar reduction in SVs at the haircell afferent synapse would also be observed in asteroid/vglut3 larvae. We performed transmission electron microscopy on hair cells from larval asteroid/vglut3 siblings and mutants.

The ultrastructure of the zebrafish hair-cell ribbon synapse is schematized in Figure 7A. A typical ribbon body is an electron-dense sphere surrounded by a filamentous halo that is decorated with synaptic vesicles. In sections that included the plasma membrane, we occasionally observed small, dense vesicular structures that appear to connect the ribbon body to the active zone (Fig. $7 B$ ).

We adopted the following criteria in scoring the number of ribbon-associated SVs: (1) a vesicle must directly appose the ribbon-halo; (2) it must be well formed (i.e., have a roughly circular cross-section); and (3) it must have a diameter of 30-50 nm (Schnee et al., 2005). These criteria exclude the recycling pool of SVs (as well as any endosomal intermediates that may happen to occur near the ribbon body) and are meant to confine our analysis only to SVs already recruited to the ribbon.

Representative sections through wild-type and asteroid/vglut3 mutant ribbons are shown in Figures $7 B(n=5$ wild-type larvae) and 7C ( $n=6$ mutant larvae), respectively. In both cases, the ribbon body is well formed and lies just above the presynaptic active zone. The halos extend 50-100 nm from the ribbon body. Although there was no difference in cross-sectional ribbon area between wild-type and mutant synapses $\left(34.4 \pm 2.1 \mu \mathrm{m}^{2}\right.$ for WT, $n=10 ; 36.8 \pm 3.4 \mu \mathrm{m}^{2}$ for asteroid/vglut3, $n=10$ ), ribbon bodies in single sections from mutant asteroid/vglut3 hair cells showed a $\sim 60 \%$ decrease in SV decoration compared with those from wild-type siblings ( $12.8 \pm 1.0 \mathrm{SVs}$ for wild-type ribbons, $n=55,5.34 \pm 0.6$; for asteroid/vglut 3 mutant ribbons, $n=56$; $p \ll 0.001$, Student's $t$ test $)$.

\section{Discussion}

In asteroid mutants, the vglut 3 gene bears a $\mathrm{T}>\mathrm{C}$ transitional mutation in the splice-donor site of intron 2 that is predicted to lead to missplicing of exon 2 during mRNA processing. The deletion of exon 2 or selection of a cryptic site within this exon results in a frameshift and premature stop codon before the first transmembrane domain, which would thus be expected to generate severely truncated Vglut 3 protein. Indeed, Vglut 3 immunoreactivity was not seen in asteroid mutants using a C-terminal antibody. These data, combined with the finding that vglut3 morpholinos replicated the asteroid mutant phenotype, demonstrate that vglut3 is identical with asteroid.

That vesicular glutamate transporters are required for hearing and balance was not unexpected given that hair cells are glutamatergic (Glowatzki and Fuchs, 2002). It is notable, however, that hearing deficits in VGLUT1 knock-out mice have not been reported (Fremeau et al., 2004). In zebrafish, both Vglut1 and Vglut3 are coexpressed in hair cells. Whether VGLUT3 is also expressed in murine hair cells remains to be determined. In the 
A

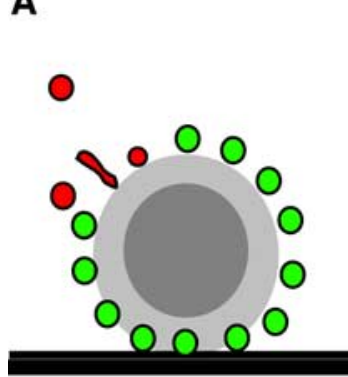

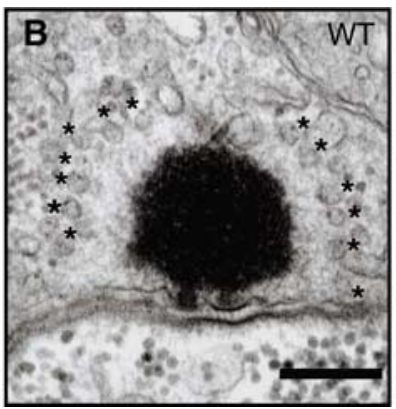

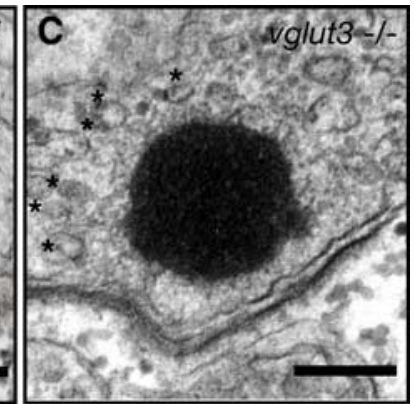

Figure 7. asteroid/vglut 3 mutants have a reduced number of ribbon-associated synaptic vesicles. $A$, Schematic of the hair cell afferent synapse. Only vesicles with a diameter of $30-50 \mathrm{~nm}$ and proximity to the ribbon were counted (green); outliers (red) were ignored. $\boldsymbol{B}$, A representative wild-type ribbon; asterisks indicate synaptic vesicles included in analysis. $\boldsymbol{C}$, A representative section through a mutant ribbon. Scale bars: $200 \mathrm{~nm}$.

mature mammalian CNS, the three VGLUT isoforms display complementary distributions, while neurons in the developing brain may transiently coexpress VGLUT1 and VGLUT2 (Fremeau et al., 2004b). Whether the presence of both Vglut 1 and Vglut3 in larval zebrafish hair cells is a transient phenomenon awaits further investigation. However, it is clear from the above results that, in contrast to immature mammalian neurons, the presence of these two Vglut isoforms is necessary in larval hair cells, as the loss of either Vglut 3 or Vglut1 alone was sufficient to render these larvae deaf.

In mammalian neurons that coexpress VGLUT1 and VGLUT2, these transporters appear to be segregated to distinct vesicle populations that traffic to separate presynaptic boutons (Fremeau et al., 2004b). We do not yet know whether the same is true of Vglut1 and Vglut3 in larval zebrafish hair cells, but Vglut3 is detectable near all ribbon bodies throughout the basal end of hair cells, suggesting that Vglut3 does not segregate to different ribbon synapses.

Our measurements of postsynaptic responses in lateral line afferent nerves indicate that synaptic transmission in asteroid/ vglut 3 mutants is severely affected. Actions currents are not detectable. This data suggests that synaptic transmission from hair cells to first order neurons does not occur, despite normal receptor potentials in these mutants. We cannot rule out that some subthreshold amount of glutamate is still released, as asteroid/ vglut 3 mutants apparently express vglut 1 at normal levels. It is evident, however, that the absence of Vglut 3 causes pronounced defects at both the behavioral and electrophysiological level in these mutants.

In mutant asteroid/vglut3 hair-cell synapses, synaptic ribbons coordinate at least $60 \%$ fewer SVs than wild-type ribbons. This result complements the work in VGLUT1 knockout mice, which display a similar reduction in SVs within 300 $\mathrm{nm}$ of the active zone at central synapses (Fremeau et al., 2004b), and implies a role for Vglut3 in the recruitment of SVs to the ribbon, or in SV recycling/biogenesis, or both. Lack of action currents suggests that Vglut3 is acting as a transporter, filling synaptic vesicles with neurotransmitter. Whether vesicle recruitment to the ribbon depends on the role of Vglut3 as a glutamate transporter per se, or is perhaps a result of physical interactions with other as yet unidentified proteins, awaits further investigation.

\section{References}

Beck JC, Gilland E, Tank DW, Baker R (2004) Quantifying the ontogeny of optokinetic and vestibuloocular behaviors in zebrafish, medaka, and goldfish. J Neurophysiol 92:3546-3561.
Deng J, Zhang F, Pang Y, Li J, Li Y (2007) Vesicular glutamate transporterimmunoreactivities in the vestibular nuclear complex of rat. Neurosci Bull 22:204-208.

Fremeau Jr RT, Troyer MD, Pahner I, Nygaard GO, Tran CH, Reimer RJ, Bellocchio EE, Fortin D, Storm-Mathisen J, Edwards RH (2001) The expression of vesicular glutamate transporters defines two classes of excitatory synapse. Neuron 31:247-260.

Fremeau Jr RT, Voglmaier S, Seal RP, Edwards RH (2004a) VGLUTs define subsets of excitatory neurons and suggest novel roles for glutamate. Trends Neurosci 27:98-103.

Fremeau Jr RT, Kam K, Qureshi T, Johnson J, Copenhagen DR, Storm-Mathisen J, Chaudhry FA, Nicoll RA, Edwards RH (2004b) Vesicular glutamate transporters 1 and 2 target to functionally distinct synaptic release sites. Science 304:1815-1819.

Furness DN, Lawton DM (2003) Comparative distribution of glutamate transporters and receptors in relation to afferent innervation density in the mammalian cochlea. J Neurosci 23:11296-11304.

Furness DN, Hulme JA, Lawton DM, Hackney CM (2002) Distribution of the glutamate/aspartate transporter GLAST in relation to the afferent synapses of outer hair cells in the guinea pig cochlea. J Assoc Res Otolaryngol 3:234-247.

Gale J, Marcotti W, Kennedy H, Kros C, Richardson G (2001) FM1-43 dye behaves as permeant blocker of the hair-cell mechanotransducer channel. J Neurosci 21:7013-7025.

Gillespie D, Kim G, Kandler K (2005) Inhibitory synapses in the developing auditory system are glutamatergic. Nat Neurosci 8: 332-338. Glowatzki E, Fuchs PA (2002) Transmitter release at the hair cell ribbon synapse. Nat Neurosci 5:147-154.

Gong J, Jellali A, Mutterer J, Sahel J, Rendon A, Picaud S (2006) Distribution of vesicular glutamate transporters in rate and human retina. Brain Res 1082:73-85.

Gras C, Vinatier J, Amilhon B, Guerci A, Christov C, Ravassard P, Giros B, El Mestikawy S (2005) Developmentally regulated expression of VGLUT3 during early post-natal life. Neuropharmacology 49:901-911.

Haverkamp S, Waessle H (2004) Characterization of an amacrine cell type of the mammalian retina immunoreactive for vesicular glutamate transporter 3. J Comp Neur 468:251-263.

Johnson J, Sherry D, Liu X, Fremeau R, Seal R, Edwards R, Copenhagen D (2004) Vesicular glutamate transporter 3 expression indentifies glutamatergic amacrine cells in the rodent retina. J Comp Neur 477:386-398.

Kazmierczak P, Sakaguchi H, Tokita J, Wilson-Kubalek EM, Milligan RA, Müller U, Kachar B (2007) Cadherin 23 and protocadherin 15 interact to form tip-link filaments in sensory hair cells. Nature 449:87-91.

Lenzi D, Crum J, Ellisman MH, Roberts WM (2002) Depolarization redistributes synaptic membrane and creates a gradient of vesicles on the synaptic body at a ribbon synapse. Neuron 36:649-659.

Lenzi K, Runyeon J, Crum J, Ellisman M, Roberts W (1999) Synaptic vesicle populations in saccular hair cells reconstructed by electron tomography J Neurosci 19:119-132.

Moser T, Beutner D (2000) Kinetics of exocytosis and endocytosis at the cochlear inner hair cell afferent synapse of the mouse. Proc Natl Acad Sci USA 97:883-888.

Nicolson T, Rusch A, Friedrich R, Granato M, Ruppersberg J, NussleinVolhard C (1998) Genetic analysis of vertebrate sensory hair cell mechanosensation: the zebrafish circlers Neuron 20: 271-283.

Schmitz F, Konigstorfer A, Sudhof TC (2000) RIBEYE, a component of synaptic ribbons: a protein's journey through evolution provides insight into synaptic ribbon function. Neuron 28:857-872.

Schnee ME, Lawton DM, Furness DN, Benke TA, Ricci AJ (2005) Auditory hair cell-afferent fiber synapses are specialized to operate at their best frequencies. Neuron 47:243-254.

Seiler C, Nicolson T (1999) Defective calmodulin-dependent rapid apical endocytosis in zebrafish sensory hair cell mutants J Neurobiol 41:424-434.

Siemens J, Lillo C, Dumont RA, Reynolds A, Williams DS, Gillespie PG, 
Müller U (2004) Cadherin 23 is a component of the tip link in hair-cell stereocilia. Nature 428:950-955.

Shu X, Shaner NC, Yarbrough CA, Tsien RY, Remington SJ (2006) Novel chromophores and buried charges control color in mFruits. Biochem 45:9639-9647.

Sollner C, Rauch GJ, Siemens J, Geisler R, Schuster SC, Muller U, Nicolson T (2004) Mutations in cadherin 23 affect tip links in zebrafish sensory hair cells. Nature 428:955-959.

Takamori S (2006) VGLUTs: "exciting" times for glutamatergic research? Neurosci Res 55:343-351.

Takumi Y, Matsubara A, Laake J, Ramiez-Leon V, Roberg B, Torgner I, Kvamme E, Usami S, Otersen O (1999) Phosphate activated glutami- nase is concentrated in mitochondria of sensory hair cells in rat inner ear. J Neurocytol 3:223-227.

Wang Y, Pang Y, Dong Y, Zhang F, Li J, Li Y (2007) Localization of vesicular glutamate transporters in the peripheral vestibular system of rat. Neurosci Bull 23:175-179.

Wojcik S, Rhee J, Herzog E, Sigler A, Jahn R, Takamori S, Brose N, Rosenmund C (2004) An essential role for vesicular glutamate transporter 1 in postnatal development nad control of quantal size. Proc Natl Acad Sci USA 101:7158-7171.

Zuber M, Strittmatter S, Fishman M (1989) A membrane-targeting signal in the amino terminus of the neuronal protein GAP-43. Nature 341:345348. 